\title{
Comparative analysis of software products for virtual simulation of electrical circuits
}

\author{
Alexey Bashkirov, Vadim Glotov ${ }^{*}$, Alexander Kostyukov, Alexander Antilikatorov, and \\ Yuri Balashov \\ Voronezh State Technical University, Russia, 394026 Voronezh, Moscow Avenue, 14
}

\begin{abstract}
This article discusses the use of special software products for the design and simulation of the operation of electrical circuits, namely, the most commonly used programs Electronic Workbench and Multisim are compared, their advantages and disadvantages are determined. In the course of the experiments, on the basis of the above presented software systems, the operation of the electrical circuits of the devices of the amplitude diode detector and the low-frequency amplifier was simulated to identify the capabilities of the programs. In the course of the research, a visual table was compiled, thanks to which it is possible to compare the obtained parameters with the reference characteristics taken from real-life devices. The uptime was chosen as a control parameter, the article contains its calculation formula, and the final calculations are given in the table to better substantiate the capabilities of the software products under consideration. At the end of the article, conclusions are presented on the work done, thanks to which it is possible to determine the pros and cons of each of the programs under consideration.
\end{abstract}

\section{Introduction}

Electronic design and development is a very important part of the modern manufacturing process. The development of the device itself is multifaceted and complex and includes many different operations, for example, the design of design documentation, virtual simulation of the device being developed, as well as the creation of a preliminary model of the device.

Reliability studies take a special place in the design of radio electronic devices. Basically, they are of several types, namely, the study of a finished device as a test sample and virtual simulation using specialized programs. For physical research, enterprises build entire laboratories with special equipment for experiments, this method is very expensive and time-consuming, but it also gives the most accurate result. As a result, only large companies can afford such research, and small and medium-sized enterprises are content with only virtual tests, we will consider them in more detail.

Currently, to conduct virtual studies for reliability, you can use many different software systems for modeling, thanks to which you can study a particular process. For example, software environments such as Creo or SolidWorks are mainly used to simulate various

*Corresponding author: vadik-livny@mail.ru 
effects on the design of electronic devices. Our research is connected with modeling the operation of electrical circuits of devices and software tools that allow carrying out such types of analyzes also exist at the present time, these include such programs as Electronic Workbench (EWB), Multisim, OrCad, Proteus, the most common of which are EWB and Multisim [1]. Both of these programs are created by the National Instruments Electronic Workbench Group, the first of which was EWB in 1989. But the Multisim software package was created only in 1999 and is a further development of the EWB system, while having a more sophisticated modeling algorithm in comparison with the previous software development.

\section{Research method}

One of the most important parameters of device reliability is the uptime, thanks to this parameter, you can evaluate the device's performance and determine its service life. This parameter can be obtained both experimentally using time-to-failure tests, and calculated using a special formula [2]:

$$
T_{o}=\frac{t(n+(n-r))}{0.0072 \mathrm{r}}
$$

where $\mathrm{t}$ - is the time spent on measurements; $\mathrm{n}$ - is the total number of all measured characteristics; $r$ - the number of characteristics that went beyond the range of normal operation.

The purpose of this study is to determine how the data obtained as a result of modeling in the EWB and Multisim programs differ from the parameters obtained during experiments on physical devices. And whether these characteristics can be used to determine the reliability of the device, in order to simplify the testing for reliability. For these purposes, simple and already well-studied devices were selected, the parameters of which are already known, and the reliability is easily determined using the above formula, to use these parameters as reference characteristics. Such devices are an amplitude diode detector and a low-frequency amplifier, electrical circuit diagrams, which are shown in Figure 1 [3].

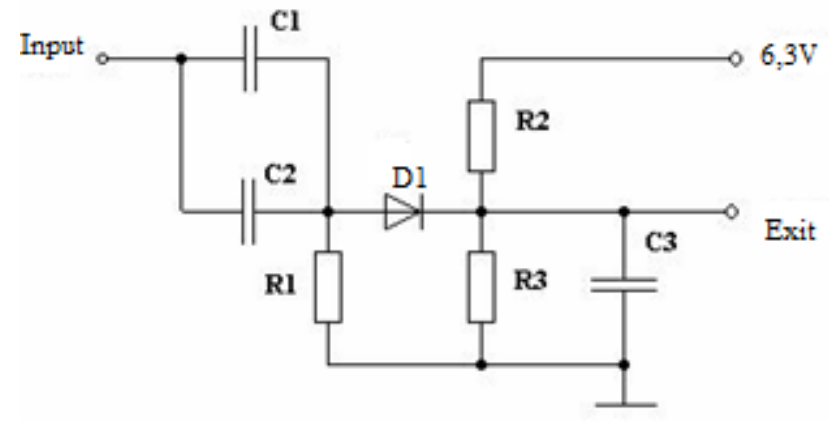

Fig. 1. Electrical schematic diagram of an amplitude diode detector

\section{The results of the measurements and calculations}

To conduct the experiment, electrical diagrams of these devices were constructed in the EWB and Multisim software environments and the frequency dependence of the output voltage was measured. The obtained parameters are compared with the reference data 
obtained during the experiment with physical device layouts. All the obtained parameters are presented in table 1 for better clarity and comparison.

Table 1. The comparison of the received data.

\begin{tabular}{|l|l|l|l|l|l|l|}
\hline \multicolumn{5}{|l|}{ Low frequency amplifier, $\mathrm{v}$} & \multicolumn{3}{l|}{ Amplitude diode detector, $\mathrm{V}$} \\
\hline $\begin{array}{l}\text { Frequency, } \\
\mathrm{Hz}\end{array}$ & $\begin{array}{l}\text { Device } \\
\text { output }\end{array}$ & Output EWB & $\begin{array}{l}\text { Output } \\
\text { Multisim }\end{array}$ & Output & $\begin{array}{l}\text { Output } \\
\text { EWB }\end{array}$ & $\begin{array}{l}\text { Outtisim } \\
\text { Multim }\end{array}$ \\
\hline 1000 & 0.003966 & 0.000382977 & 0.003970 & 4.2385 & 4.1012 & 4.2389 \\
\hline 2000 & 0.003798 & 0.000184523 & 0.0038 & 4.2383 & 4.2560 & 4.2386 \\
\hline 3000 & 0.00356 & 0.000114495 & 0.003601 & 4.2376 & 4.3361 & 4.238 \\
\hline 4000 & 0.003299 & 0.000078552 & 0.003302 & 4.2376 & 4.2354 & 4.2375 \\
\hline 5000 & 0.003045 & 0.000056987 & 0.003053 & 4.2376 & 4.2362 & 4.2372 \\
\hline 6000 & 0.002813 & 0.000042936 & 0.002826 & 4.2378 & 4.24 & 4.2377 \\
\hline 7000 & 0.002608 & 0.000033888 & 0.002611 & 4.2378 & 4.2363 & 4.2381 \\
\hline 8000 & 0.002431 & 0.000027638 & 0.002425 & 4.2379 & 4.2391 & 4.2387 \\
\hline 9000 & 0.002278 & 0.000023065 & 0.002281 & 4.2385 & 4.2377 & 4.2387 \\
\hline 10000 & 0.002148 & 0.000019588 & 0.002139 & 4.2385 & 4.2386 & 4.2390 \\
\hline 11000 & 0.002037 & 0.000016882 & 0.002021 & 4.2392 & 4.2395 & 4.2389 \\
\hline 12000 & 0.001941 & 0.000014773 & 0.001957 & 4.2388 & 4.2405 & 4.2387 \\
\hline 13000 & 0.001858 & 0.000013079 & 0.001841 & 4.2386 & 4.2381 & 4.2385 \\
\hline 14000 & 0.001787 & 0.000011691 & 0.001775 & 4.2381 & 4.2372 & 4.2379 \\
\hline 15000 & 0.001724 & 0.000010545 & 0.001718 & 4.2379 & 4.2368 & 4.2377 \\
\hline 16000 & 0.00167 & 0.000009584 & 0.001662 & 4.2375 & 4.2501 & 4.2374 \\
\hline 17000 & 0.001622 & 0.000008775 & 0.001619 & 4.2375 & 4.2352 & 4.2373 \\
\hline 18000 & 0.00158 & 0.000008081 & 0.001587 & 4.2376 & 4.2383 & 4.2375 \\
\hline 19000 & 0.001543 & 0.000007483 & 0.001538 & 4.2380 & 4.2374 & 4.2381 \\
\hline 20000 & 0.00151 & 0.000006958 & 0.001491 & 4.2385 & 4.2358 & 4.2387 \\
\hline 21000 & 0.00148 & 0.000006506 & 0.001472 & 4.2385 & 4.2172 & 4.2386 \\
\hline 22000 & 0.001453 & 0.000006097 & 0.001449 & 4.2386 & 4.2285 & 4.2387 \\
\hline 23000 & 0.001429 & 0.000005746 & 0.001425 & 4.2389 & 4.2335 & 4.2391 \\
\hline 24000 & 0.001407 & 0.000005425 & 0.001401 & 4.2385 & 4.2406 & 4.2388 \\
\hline 25000 & 0.001387 & 0.000005138 & 0.001395 & 4.2379 & 4.2291 & 4.2380 \\
\hline 26000 & 0.001369 & 0.000004881 & 0.001365 & 4.2374 & 4.2387 & 4.2377 \\
\hline 27000 & 0.001353 & 0.000004641 & 0.001357 & 4.2373 & 4.2356 & 4.2375 \\
\hline 28000 & 0.001338 & 0.000004432 & 0.001341 & 4.2373 & 4.2348 & 4.2374 \\
\hline 29000 & 0.001324 & 0.000004234 & 0.001329 & 4.2377 & 4.2335 & 4.2378 \\
\hline 30000 & 0.001311 & 0.000004056 & 0.001302 & 4.2387 & 4.2255 & 4.2386 \\
\hline & & & & & & \\
\hline
\end{tabular}

After analyzing Table 1, you can come to some conclusions, so the most accurate data, close to the values that were obtained in the course of studying physical devices, are the characteristics obtained as a result of circuit simulation in the Multisim software package. These parameters begin to diverge from the control characteristics only after $10^{-5}$ sign; for further mathematical calculations, the resulting error is insignificant. The characteristics obtained thanks to the EWB program are very different from the control measurements of physical devices, some of them at the same measurement frequency do not coincide at all and are different, in this regard, these parameters during further mathematical calculations may show an unreliable solution. The result obtained can be explained by the fact that the Multisim software package is a further development of the EWB, as noted above, and as a result has a more perfect algorithm for modeling processes, in which many shortcomings of the previous version are corrected.

The next step of the study is to find the uptime for the above characteristics according to formula and compare how much the final answer depends on the initial data obtained in 
EWB and Multisim in comparison with experimental data, so as a result of calculations, the following expressions were obtained, given in the table 2 .

Table 2. Time between failures

\begin{tabular}{|l|l|c|c|c|c|c|}
\hline \multirow{2}{*}{} & \multicolumn{3}{|c|}{ Low frequency amplifier } & \multicolumn{3}{c|}{ Amplitude diode detector } \\
\cline { 2 - 7 } & Model & EWB & Multisim & Model & EWB & Multisim \\
\hline $\mathrm{T}$ & 35000 & 17777.8 & 35000 & 1016670 & 1016670 & 1016670 \\
\hline
\end{tabular}

After analyzing Table 2, one can make sure that the characteristics obtained as a result of modeling in the Multisim software package are the most similar to the control parameters, since ultimately the uptime calculated from these data does not differ from the uptime calculated from the data obtained in the course of research of physical models of devices. And as noted above, the difference in characteristics obtained as a result of simulating the electrical circuits of devices in the EWB played a bad role, the uptime of the low frequency amplifier differs from the reference value.

\section{Conclusion}

In this article, a comparative analysis of the EWB and Multisim software products was carried out, with the help of which it is possible to perform a virtual simulation of electrical circuits. In the course of the research, it was revealed that the best system for this type of modeling is the Multisim software product, since the values obtained with this program are the most similar to the values that can be obtained during testing of physical devices, compared to the EWB software package. And as a consequence, the further calculation of the parameters of interest will be carried out most correctly with the smallest error.

\section{References}

1. A.S. Kostyukov, I.S. Bobyl, L.N. Nikitin, A.A. Pirogov, Analysis of statistical test methods and optimization of the calculation of reliability in the production of electronic tools, Bulletin of the Voronezh State University, v.14(3), pp. 108-114 (2018).

2. L.N. Kechiev, Design of Printed Circuit Boards for High-speed Digital Equipment (Moscow, 2007)

3. M.A. Romashchenko, The Main Tasks of the EMC Support Analysis in the Radioelectronic Equipment Design and the Principles of its Implementation, Bulletin of the Voronezh State Technical University, v. 7(4)., pp. 106-109. (2016). 